\title{
Empirical Analysis of Wireless Body Sensor Systems with Multipath Channels
}

\author{
Woosik Lee, Jina Hong and Namgi Kim* \\ Department of Computer Science, Kyonggi University \\ Iui-dong, Yeongtong-gu, Suwon-si, Gyeonggi-do, Korea \\ \{wslee,jin-a,ngkim\}@kgu.ac.kr
}

\begin{abstract}
In wireless body sensor system, various multipath environments can be established because sensor nodes can be moved to many locations as it is deployed in parts of the human body. This study compared and analyzed changes of the received signal strength (RSS) in various experimental locations, such a ground site, corridor, and room, as an actual sensor device is attached to a human body. Experimental results showed that the room, which contained many multipaths, exhibited the best RSS. The ground site, which had the lowest number of multipaths, registered the worst RSS. On the basis of these findings, we conclude that the design of body sensor systems should consider the presence of multipaths in various experimental locations.
\end{abstract}

Keywords: body sensor network; sensor network; multipath channel; cclo00 radio module; RSSI

\section{Introduction}

Sensor networks, which were initially studies on the basis of wireless sensor networks (WSNs), are currently being developed into wireless body sensor network systems (WBSNSs) [1]. In an existing WSN environment, sensor nodes perform routine jobs in a static environment so that only the minimum requirements for configuring protocol or energy management are taken into account. By contrast, a WB-SNS environment features a sensor node attached to a part of the human body, necessitating consideration of signal changes due to human body structure, body movement, sensor placement, and the characteristics of the current environment $[2,3]$. Attaching a sensor inside a human body requires compact batterybased operation, giving rise to the issue of energy management requirement that has not been incorporated into existing sensor network environments. The channel conditions necessary for a sensor node attached to a human body periodically changes in accordance with body movement; thus an energy management method suitable for a dynamic environment is required. In our previous work [2], we conducted an experiment in which sensors were attached to various parts of a human body in a standing position. This experiment was intended to analyze the effects of different human body parts and transmission power levels (TPLs) on the effectiveness of the sensors, but the influence of the established experiment environment was disregarded, so that the effects of the overall environment of the body sensor network could not be analyzed. In the present research, therefore, we used a CC1000 radio module [4], which has been extensively employed in WB-SNS research. This module

* Corresponding author: Namgi Kim 
was used to enable the consideration of various multipath environments, as well as to measure the received signal strength (RSS) at various locations, such as a ground site, corridor, and room. The measurement was implemented on the basis of various TPLs and sensor placements in various environments.

\section{Related Work}

A sensor device in WB-SNS can have various multipaths, depending on its location. Thus, even sensor devices that are deployed in the same location in a body can generate different results that correspond to the location of the devices. Previous studies on sensor networks disregard surrounding locations given that most sensors perform a specific task only at a fixed placement [5-7]. Therefore, the direct application of existing technologies for sensor network environments to WB-SNSs is constrained because of body structure, body movement, and sensor placement. Conclusively, channel analysis of various environments should be carried out to enable the efficientconstruction of WB-SNSs. Natarajan et al. [8] conducted an experiment using 12 sensor devices at 3 different environments with 14 volunteers to analyze a WB-SNS channel. Their study was limited in that it could not comprehensively analyze the effects of multipaths; this deficiency is attributed to the failure of the authors to consider an authentic environment, i.e., an actual location where individuals move. Lee et al. [2] also experimentally analyzed various sensor placements at a fixed location. Despite the contributions of this research, it suffered from inadequate consideration of multipaths shortcoming that originated from the focus on sensor placement and neglect of the characteristics of human-based locations. Other studies have been devoted to analyzing the radio propagation of chips that are widely used in sensors $[9,10]$, but such explorations examine this issue only with respect to WSNs. As a result, whether other types of propagation may be generated upon sensor deployment to actual WB-SNSs cannot be ruled out. To construct a system where WB-SNS environments are taken into consideration, actual sensor devices should be deployed to parts of a human body and then the environment where various multipaths exist should be analyzed. On the basis of the examination, WB-SNS designers can construct various transmission power control algorithms to increase the energy efficiency in sensor nodes [11-14]. In the current work, sensors were deployed in many different parts of a human body and RSS measurement experiments were conducted in ground, corridor, and room environments. The results are expected to advance methods of satisfying the aforementioned requirements.

\section{Data Format and Protocol}

An experiment was conducted, in which a new structure was added on the basis of the basic data format provided by TinyOS. The basic active message format of TinyOS consists of the Destination, Active Message handler ID, Group ID, Message length, and Payload, generating $2,1,1,1,1$, and 29 byte sizes, respectively, amounting to a total of 32 bytes of a message format. The data structure in the Payload was modified (Figure 1) for data collection and sensor initialization. The data structure consists of five frames: the Beacon, GetLog, Report, Setting, and Reset Frames. Beacon is a frame that is periodically sent by a Sink Node that consists of the Sequence Number of the transmission frame sent by the current Sink Node, Sink Node ID, and the Power Level of the transmission frame sent by the current Sink Node. GetLog is a frame for the collection of log data on the Sensor Node sent by a Gateway. Report is a frame sent by a Sensor Node as soon as it receives the GetLog Frame from the 
Gateway node. It consists of its own current sensor ID, as well as a Sequence Number, Power Level, and RSS Indication (RSSI) received from the Beacon Frame. The Setting Frame is used to signal the initiation of the Beacon Message transmission and Power Level Setting of a Sink Node. The Reset message is responsible for moving the Log data of a Sensor Node to the initialization point, as well as for initialization.

\begin{tabular}{|c|c|c|c|c|c|}
\hline \multicolumn{5}{|c|}{ General MAC Frame Message Format } & \\
\hline Octets: 2 & 1 & 1 & 1 & 29 & \\
\hline Destination Addresss & Handler ID & $\begin{array}{c}\text { Group } \\
\text { ID }\end{array}$ & Message Length & Payload & \\
\hline \multicolumn{6}{|l|}{ Beacon Frame } \\
\hline Bits: 1 & 1 & 1 & 1 & 1 & 3 \\
\hline Sink Node ID & Sensor Node ID & SEQ & Power Level & Rssi & Reserved \\
\hline \multicolumn{6}{|l|}{ GetLog Frame } \\
\hline \multicolumn{6}{|l|}{ Bits: 1} \\
\hline \multicolumn{6}{|l|}{ Sensor Node ID } \\
\hline \multicolumn{6}{|l|}{ Report Frame } \\
\hline Bits: 1 & 1 & 1 & 1 & 1 & 3 \\
\hline Sink Node ID & Sensor Node ID & SEQ & Power Level & Rssi & Reserved \\
\hline \multicolumn{6}{|l|}{ Setting Frame } \\
\hline Bits: 1 & 1 & & & & \\
\hline Sink Node ID & Power Level & & & & \\
\hline \multicolumn{6}{|l|}{ Reset Frame } \\
\hline Bits: 1 & & & & & \\
\hline Dump & & & & & \\
\hline
\end{tabular}

\section{Figure 1. MAC Frame Format in WB-SNSs}

The experiment was carried out via four procedures: channel information saving, channel information collection, sink setting, and sensor initialization. These procedures enabled the analysis of the channels over a WB-SNS environment. Figure 2 shows the message sequence chart for channel information saving. A Sink Node transmits the Beacon Frame at every $100 \mathrm{~ms}$ to the Sensor Node using multicast, while a Sensor Node stores the current RSSI value and information kept in the Beacon Frame into EEPROM.

Figure 3 illustrates the message sequence chart for channel information collection. To perform channel information collection, a user's PC requests Log data collection from the Gateway Node, which then transmits the GetLog Frame to a specific sensor by using unicast. After this step, the Sensor Node that receives the GetLog Frame transmits the log information stored in its own EEPROM to the Gateway Node by piggybacking it in the Report Frame. The Gateway Node subsequently transmits the Report Frame to the user's PC. Finally, the PC stores the channel information that is received from the Gateway Node for analysis.

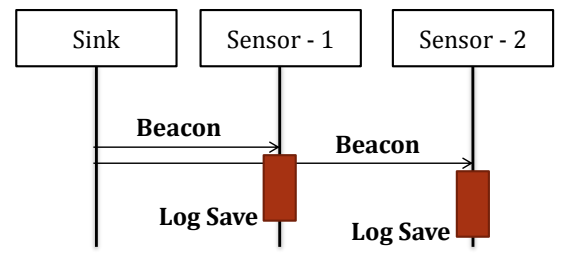

Figure 2. Data Message Sequence Chart for Logging Data 


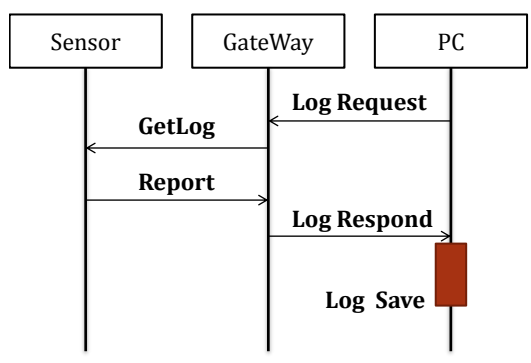

Figure 3. Message Sequence Chart for Getting Log Data

Figure 4 presents the Message Sequence Chart for initializing the Sink Node. A user's PC transmits the initialization request message to the Gateway Node for initialization. Here, the Gateway Node transmits the Setting Frame to the Sink Node with unicast. The Sink Node that receives the Setting Frame initializes the Sequence Number and current Power Level. The Sink Node then counts message sequence for the storage of Beacon Node channel information after the period set on a timer expires.

Figure 5 shows the Message Sequence Chart for sensor initialization. The sensor initialization request is an event that occurs after the Gateway node collects the Log data of all surrounding Sensor Nodes. Thus, a user's PC periodically verifies whether the Log data of all Sensor Nodes around the Gateway Node are collected. Once the log data collection is completed, the Reset request message is transmitted to the Gateway Node for sensor initialization. The Gateway Node that receives the Reset request message delivers the Reset Frame to all sensors by using multicast. Then, the Sensor Nodes that receive the Reset Frame set their Log pointer to the initialization value to perform initialization for all values.

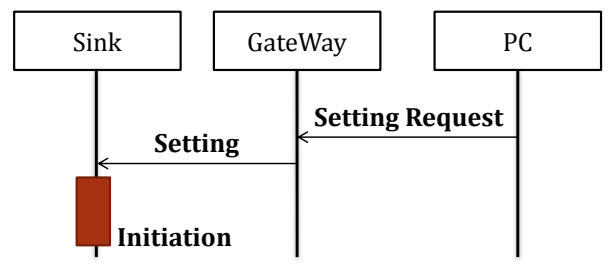

Figure 4. Message Sequence Chart for Sink Node Initiation

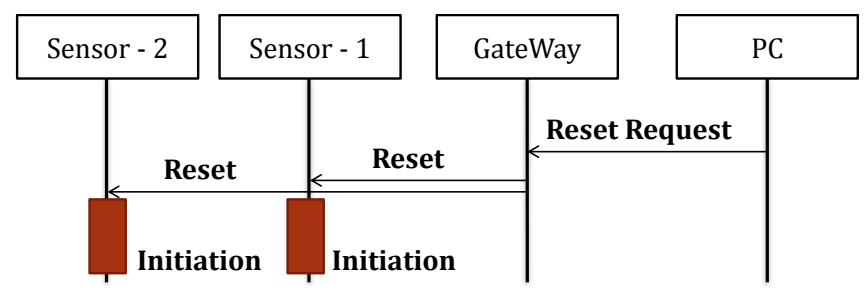

Figure 5. Message Sequence Chart for Sensor Node Initiation 


\section{Experimental Environment}

Table 1. Properties of Experimental Mote

\begin{tabular}{|c|c|}
\hline Properties & Description \\
\hline Platform & Cricket Mote \\
\hline Radio Chip & CC1000 Radio Chip \\
\hline Power Level Rang & $1 \sim 22$ (total 22 levels) \\
\hline Frequency & $868 \mathrm{MHz}$ \\
\hline Power Control & Programmable \\
\hline RF Module & Zigbee \\
\hline Supply Voltage & 2.1 to 3.6V \\
\hline Transmit Bit Rate & $115.2 \mathrm{Kbps}$ \\
\hline
\end{tabular}

Another experiment was that in which Cricket Mote sensors were deployed in the arm, stomach, and back of an individual (Figure 6) to analyze accurate channels over a body sensor system environment. The Sink Node was deployed in the chest while the Gateway Node was connected to a notebook. Table 1 shows the properties of the sensor device used in the experiment. The platform of the device used in the experiment was Cricket Mote [16] provided by MEMSIC [15]; a CC1000 Radio Chip [4] was mounted in Cricket Mote. The CC1000 Radio Chip has 22 TPLs, which can be freely varied. The frequency band of the CC1000 Radio chip is $868 \mathrm{MHz}$ and its transmit bit rate is $115.2 \mathrm{Kbps}$. The power supply for Cricket Mote uses two 1.5 V AA batteries. Five Cricket Motes were used for this experiment.

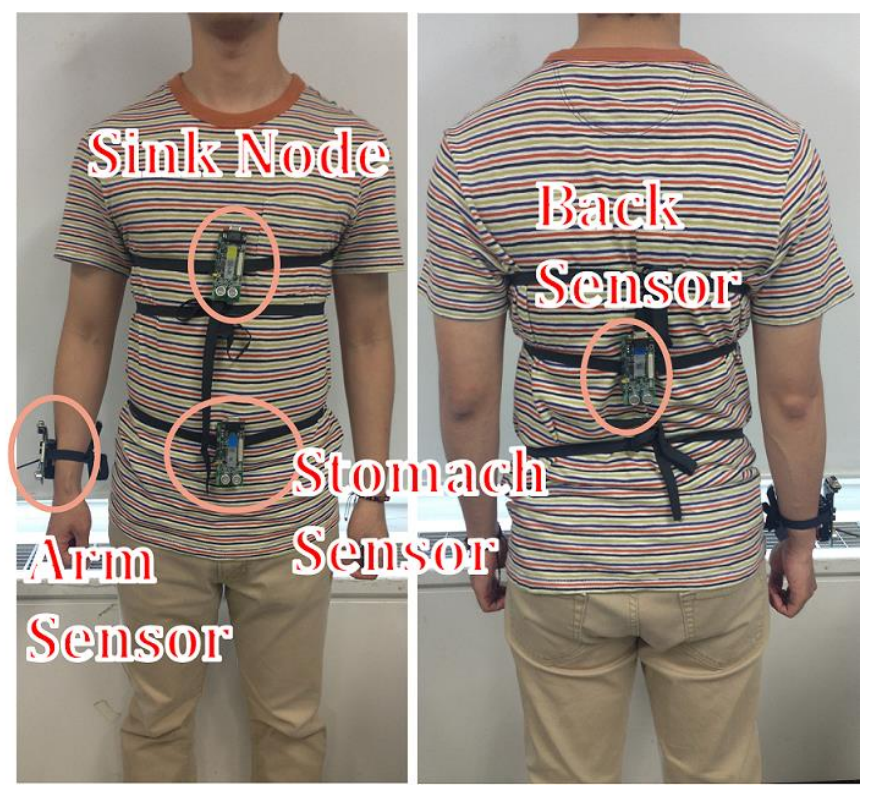

Figure 6. Sink Node and Sensor Node Deployment on the Body

Figure 7 shows the location at which the experiment was conducted for the analysis of channels over the WB-SNS environment. We chose three locations for the analysis: a ground site where a few multipaths exist, a corridor where a few multipaths also exist, and a room where many multipaths are present because of numerous obstacles. 


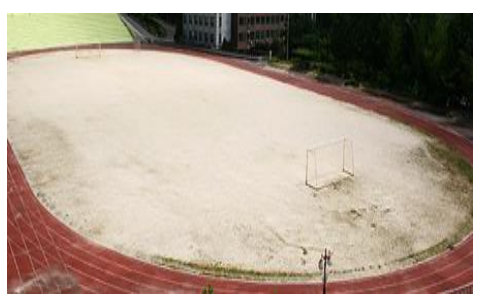

Ground

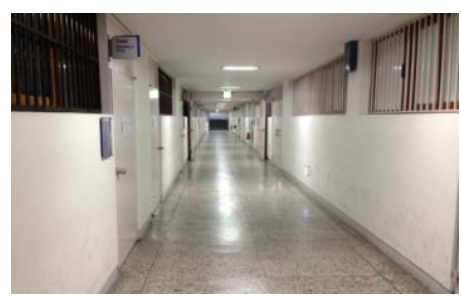

Corridor

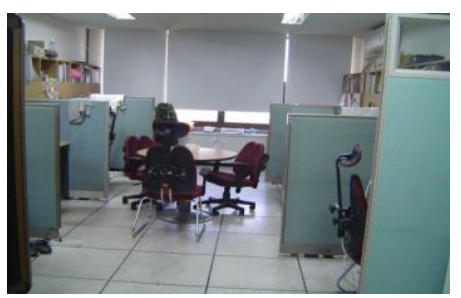

Room

Figure 7. Sink Node and Sensor Node Deployment on the Body

\section{Experimental Result}

The RSSI, Power Level, and packet delivery rate (PDR) were taken into consideration as the elements for the analysis. The RSSI is a received power strength value that reflects current channel conditions, while Power Level is an element that disrupts communication distance and interference. Figure 8 shows the RSSI values generated when packets were sent to the Sensor Node placed on the stomach as a Sink Node set the TPL to 10 and 22. The findings showed that the stomach sensor, which is the closest to the Sink Node, exhibited no significant difference in RSSI values, regardless of location change. This result is attributed to the fact that no obstacle was found between the Sink Node and the Sensor Node, thereby maintaining line-of-sight (LOS). Thus, the stomach Sensor Node was rarely affected by the multipaths generated in the different experimental locations.

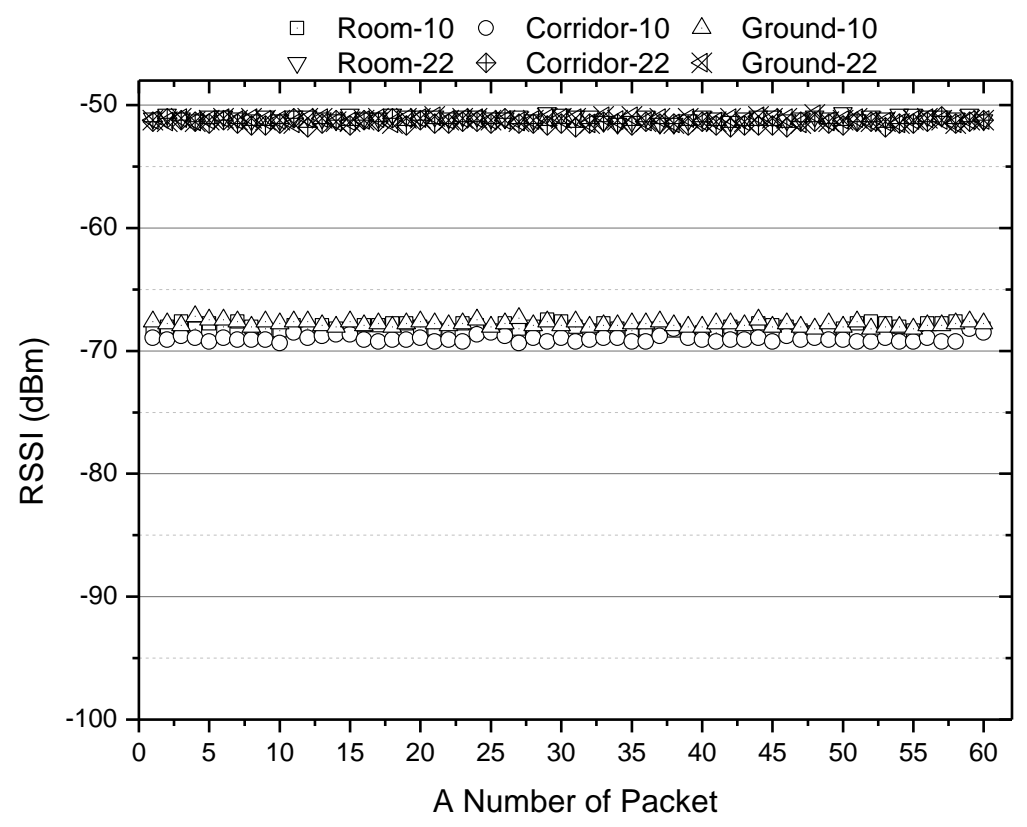

Figure 8. RSSI Values of the Stomach for Different Experimental Locations

Figure 9 indicates the RSSI value of the Sensor Node placed on the arm under the same experimental setting values in Figure 8 . The RSSI value slightly changed in accordance with location, in contrast to the results shown in Figure 8. In particular, the ground site exhibited the worst RSSI value, whereas the room produced the best RSSI. 
This result was due to the blocked walls of the room in all directions; as the Sink Node transmitted data, therefore, numerous multipaths were generated given signal reflection. In the ground site, where no blocking obstacle was found, the signal reflection effect rarely occurred; thus, a low RSSI value given the small path that received the signal. The corridor exhibited results similar to those for the room because the walls on both sides of the former easily created signal reflection. On the basis of these results, we found that sensor placement is unaffected by location if a sink node is established as the LOS. Conversely, if a sensor is not placed along the LOS, the RSSI is significantly affected by location. Overall, the RSSI value of the arm was lower than that of the stomach because of the signal attenuation that stemmed from the blocking or shadowing of radio signals by the obstacles in the surrounding environment.

Figure 10 shows the RSSI value of the sensor located at the back of the individual being tested under the same setting values shown in Figures 8 and 9. The RSSI values of the ground and corridor were generally lower than those of the stomach and arm. This difference is attributed to the long distance that was generated between the path and the Sensor Node placed at the back as the Sink Node transmitted data. By contrast, the result for the room did not significantly decrease compared to those for the other two locations, which exhibited the highest RSSIs. This discrepancy is attributed to the attenuation of signal strength due to reflection by the walls; this reflection was compensated by the numerous multipaths in the room.

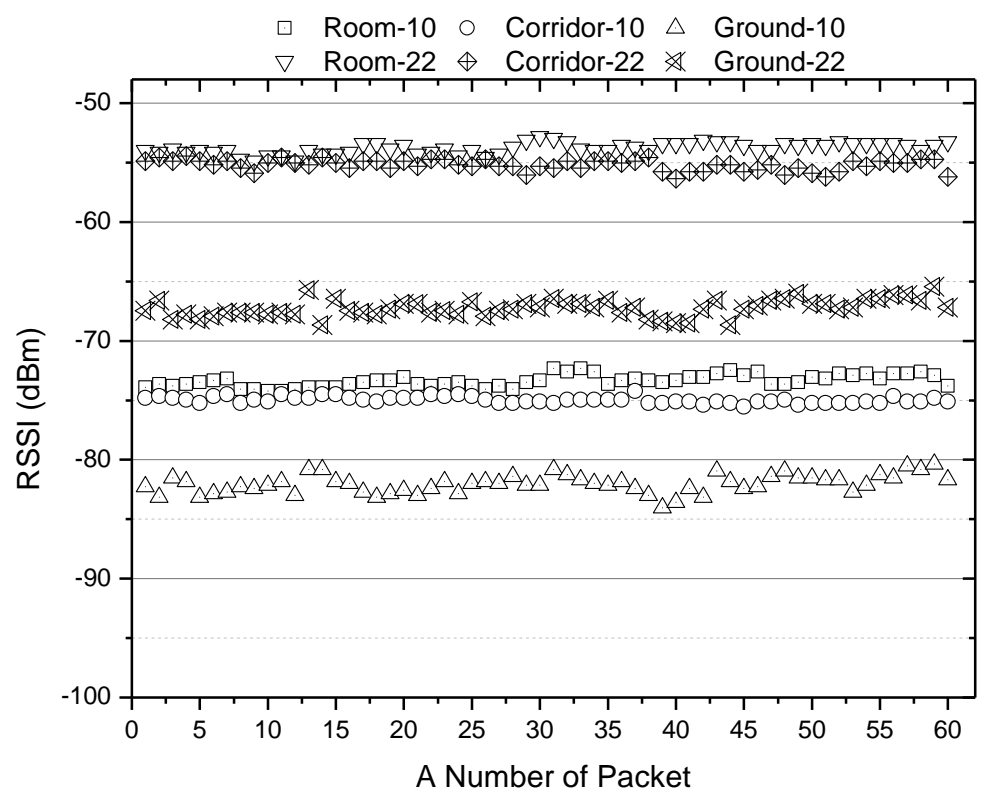

Figure 9. RSSI Values of the Stomach for Different Experimental Locations 


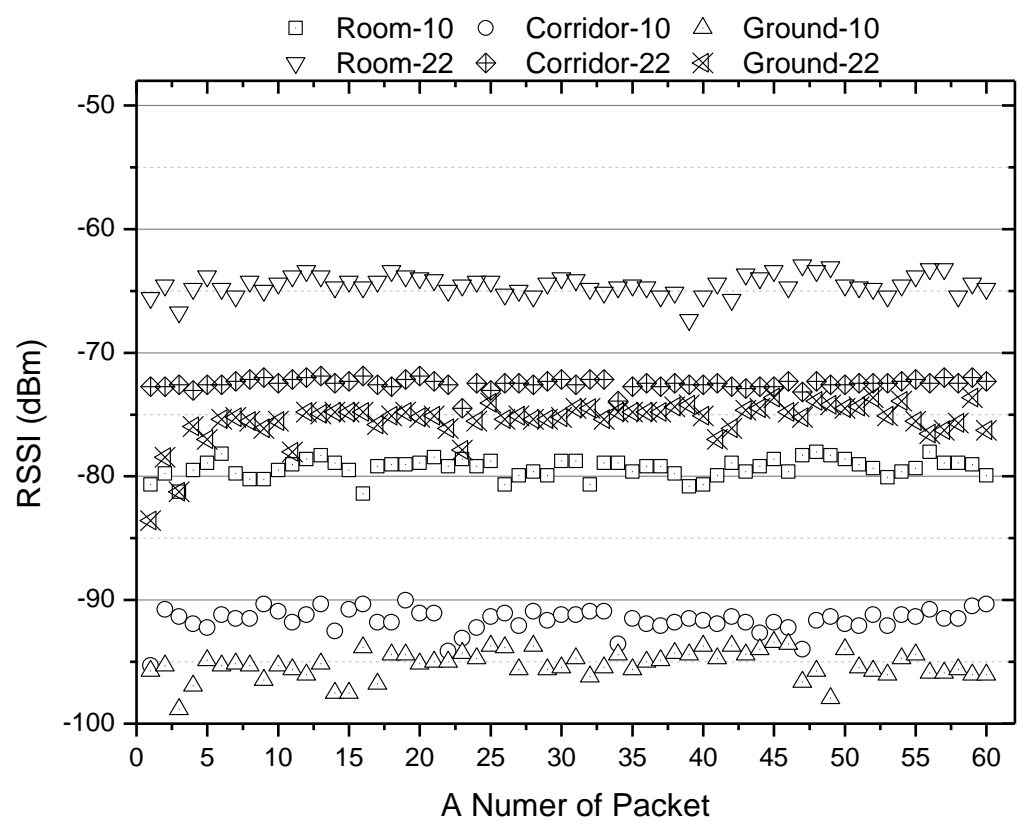

Figure 10. RSSI Values of the Back for Different Experimental Areas

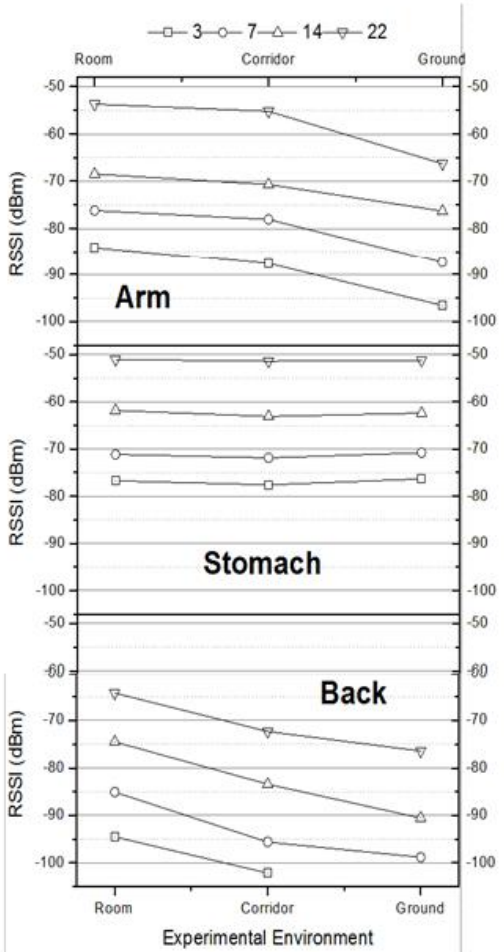

Figure 11. Average RSSI Values of the Stomach, Arm, and Back for Different Experimental Locations

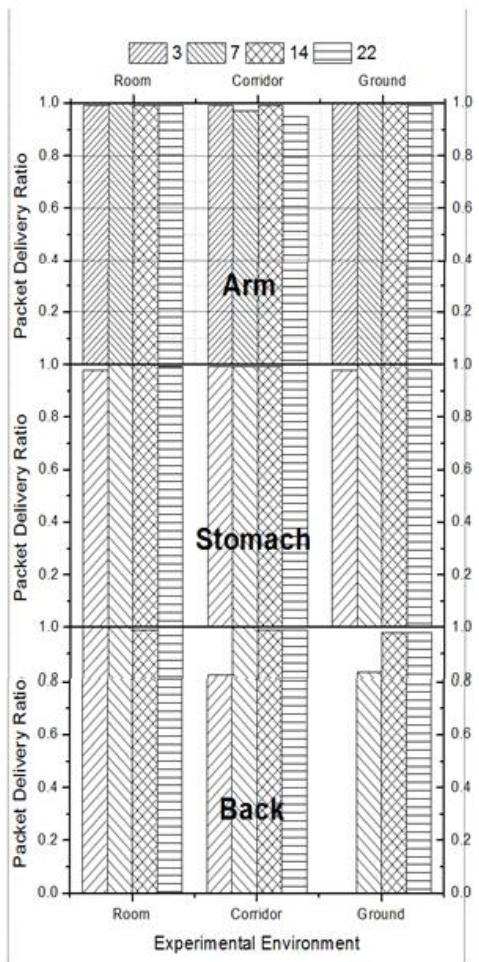

Figure 12. Average RSSI Values of the Stomach, Arm, and Back for Different Experimental Locations 
Figure 11 shows the average RSSI graph, derived using 3, 7, 14, and 22 TPLs in the different experimental locations. The upper graph shows the result for the Sensor Node deployed in the arm, while the middle and lower graphs present the findings for the stomach and back, respectively. The $\mathrm{x}$-axis of the graph indicates the experimental location and the y-axis refers to the average RSSI value. First, the higher the TPL, the better the RSSI value in terms of power level. That is, an increase in TPL translated to an improvement in the communication channel. Second, the average RSSI value worsened in the order room > corridor > ground in terms of experimental location. These results were clear in the case of the arm and back placements, but not for the stomach placement where the LOS was found. Furthermore, the back placement at Level 3 in the ground generated no RSSI value because the Sensor Node did not receive data from the Sink Node.

Figure 12 presents the PDR, determined according to the placement of the Sensor Nodes. The $\mathrm{x}$-axis of the graph represents the experimental location and the $\mathrm{y}$-axis represents the PDR. The Sensor Nodes placed in the stomach and arm maintained a PDR greater than 95\%, whereas the Sensor Node placed in the back exhibited a decreasing PDR at low TPLs of the Sink Nodes in the corridor and ground, where multipaths occurred relatively less frequently. At a TPL of 3 for the ground scenario, no data was received by the Sensor Node from the Sink Node.

This study showed results that varied, depending on sensor placement and experimental location. The room, where the multipath effect most frequently occurred, produced the best result, whereas the corridor, where the multipath effect moderately occurred, exhibited a result worse than that generated by the room. Moreover, the ground, where the multipath effect rarely took place, presented the worst result. On these bases, we conclude that the effective construction of a body sensor system necessitates the consideration of the multipath effect and sensor placement.

\section{Conclusion}

In this study, an experiment for the analysis of various channels was conducted in accordance with the multipaths in a body sensor system. A CC1000 Radio Module, which has been widely used in actual body sensor network systems, was used. The experimental locations chosen as multipath environments were a room, corridor, and ground site. The room, which had the highest number of multipaths exhibited better results than did the ground site. The findings indicated that the multipaths complemented the communication between the Sink and Sensor Nodes. Therefore, taking the multipath effect into account in the design of body sensor systems will result in systems that are characterized by high energy efficiency and the configuration of good channel usage. Our future research will involve a study on an energy-efficient mechanism that can adaptively react to the real multipaths used in the current study. 


\section{Acknowledgements}

This research was supported by Basic Science Research Program through the National Research Foundation of Korea (NRF) funded by the Ministry of Education, Science and Technology (grant number 2012R1A1A1002133).

\section{References}

[1] M.A Hanson and et al., "Body Area Sensor Networks: Challenges and Opportunities", IEEE Computer, vol. 42, (2009) January, pp. 58-65.

[2] W. S. Lee, M. Choi and N. Kim, "Experimental link channel characteristics in wireless body sensor systems", ICOIN, (2012) February, pp. 374-378.

[3] W. S. Lee, M. Choi and N. Kim, "Different Characteristics of Radio Modules in Wireless Body sensor network systems", NPC, (2012).

[4] CC1000 Data Sheet, http://www.ti.com/lit/ug/swru058/swru058.pdf.

[5] G. N. Purohit, S. Verma and M. S. Harma, "Sensing and Communication Energy Consumption in Static Sensor Network", IJCA, vol. 17, (2011) March, pp. 13- 20.

[6] R. Szewczyk, A. Mainwaring, J. Anderson and D. Culler, "An analysis of a large scale habit monitoring application", SenSys, (2004).

[7] D. Tian and N. D. Georganas, "A node scheduling scheme for energy conservation in large wireless sensor networks", Wireless Communications and Mobile Computing Journal, (2003).

[8] A. Natarajan, B. de Silva, K. K. Yap and M. Motani, "Link Layer Behavior of Body Area Networks at 2.4 GHz", MobiCom, (2009) September, pp. 241-252.

[9] S. H. Ahmed, S. H. Bouk, N. Javaid and I. Sasase, "RF propagation analysis of MICAz Mote's antenna with ground effect", INMIC, (2012) December, pp. 270-274.

[10] D. Balachander, et al., "RF Propagation Experiments in Agricultural Fields and Gardens for Wireless Sensor Communications", PIERC, (2013) March, pp. 103-118.

[11] W. S. Lee and N. Kim, "Comparisons of Transmission Power Control Algorithms in Wireless Body Sensor Systems”, IJMUE, vol. 8, (2013) July, pp. 303-312.

[12] W. Lee, M. Choi and N. Kim, "A Study of Dynamic Transmission Power Control Algorithm in Wireless Body Sensor Network Systems", Proceedings of Advanced Science and Technology Letters, vol. 25, (2013) July, pp. 9-12.

[13] J. Zhou, X. Zhang, M. Jacobsson and I. Niemegeers, "Link quality-based transmission power adaptation for energy saving in IEEE 802.11", PIMRC, (2009), pp. 52-56.

[14] M. Meghji and D. Habibi, "Transmission Power Control in Multihop WirelessSensor Networks", ICUFN, (2011), pp. 25-30.

[15] MEMSIC Co., http://www.memsic.com.

[16] Cossbow-Technology, http://bullseye.xbow.com:81/index.as.

\section{Authors}

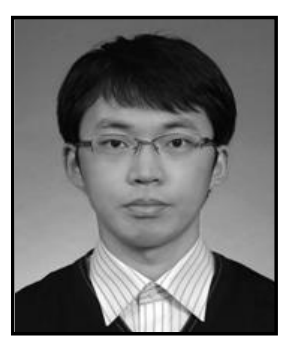

\section{Woosik Lee}

He received the B.S. degree in Computer Science from the Kyonggi University, Korea, in 2009, and the M.S. degree in the Computer Science from Kyonggi University in 2011. He is currently Ph.D. candidate in Computer Science from Kyonggi University. His research interests include wireless systems, sensor networks, and energy management protocols. 


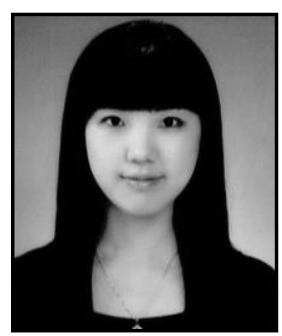

\section{Jina Hong}

She received the B.S. degree in Computer Science from the Kyonggi University, Korea, in 2013. She is currently M.S. candidate in Computer Science from Kyonggi University. Her research interests include wireless systems, sensor networks.

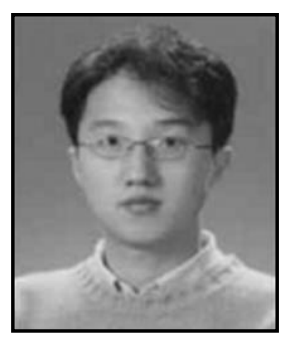

\section{Namgi Kim}

He received the B.S. degree in Computer Science from Sogang University, Korea, in 1997, and the M.S. degree and the Ph.D. degree in Computer Science from KAIST in 2000 and 2005, respectively. From 2005 to 2007, he was a research member of the Samsung Electronics. Since 2007, he has been a faculty of the Kyonggi University. His research interests include sensor system, wireless system, and mobile communication. 
International Journal of Bio-Science and Bio-Technology Vol.6, No.1 (2014) 